\title{
A Set of Novel Microsatellite Markers Developed for Luculia yunnanensis (Rubiaceae), an Endangered Plant Endemic to Yunnan, China
}

\author{
Hong Ma ${ }^{1}$, Lan Wang ${ }^{2}$, Youming Wan ${ }^{1}$, Hongzhe $\mathrm{Li}^{3}$, Zhenghong Li ${ }^{1}{ }^{*}$, Xiuxian Liu ${ }^{1}$, \\ Ning Liang ${ }^{1}$ and Wenjuan $\mathrm{Li}^{1}$
}

1 Research Institute of Resource Insects, Chinese Academy of Forestry, Kunming 650224, China; E-Mails: mahongmh@gmail.com (H.M.); wanyouming@126.com (Y.W.); 1xx205@126.com (X.L.); ln417@126.com (N.L.); hanbing2587cn@yahoo.com.cn (W.L.)

2 Yunnan Reascend Tobacco Technology (Group) Co., Ltd., Kunming 650106, China; E-Mail: 1wang0124@gmail.com

3 Faculty of Traditional Chinese Pharmacy, Yunnan University of Traditional Chinese Medicine, Kunming 650500, China; E-Mail: lihongzheyn@163.com

* Author to whom correspondence should be addressed; E-Mail: hortscience@163.com; Tel.: +86-871-3860032; Fax: +86-871-3860027.

Received: 31 October 2011; in revised form: 23 December 2011 / Accepted: 28 December 2011 / Published: 4 January 2012

\begin{abstract}
The genus Luculia Sweet contains about five species of small trees or shrubs and is a member of the family Rubiaceae (tribe Cinchoneae). Luculia yunnanensis is an endangered ornamental shrub endemic to southwest China. Only two natural populations of L. yunnanensis exist in the wild according to our field investigation. It can be inferred that L. yunnanensis is facing a very high risk of extinction in the wild and an urgent conservation strategy is required. By using a modified biotin-sterptavidin capture method, 24 primer sets were identified in two wild populations. Of these primers, 11 displayed polymorphisms and 13 were monomorphic. The number of alleles per locus ranged from two to four, values for observed and expected heterozygosities ranged from 0.000 to 0.833 and from 0.431 to 0.771 , with averages of 0.389 and 0.614 , respectively. These markers will be useful for further investigation of conservation of resources, selecting parental types in cross-breeding, evolution of this species at the molecular level and related research in Luculia species.
\end{abstract}


Keywords: genetic diversity; Luculia yunnanensis; microsatellite marker; polymorphism; population structure; Rubiaceae

\section{Introduction}

The genus Luculia Sweet contains about five species of small trees or shrubs and is a member of the family Rubiaceae (tribe Cinchoneae) [1]. It can be easily recognized by its beautiful pink, sweetly fragrant and long-term blooming time flowers, and therefore has been horticulturally introduced into various regions and botanical gardens around the world. Three Luculia species exist in China; among them L. yunnanensis $\mathrm{Hu}$ is the unique one which is endemic to Yunnan Province, naturally confined to forests or thickets on limestone mountains, open slopes and secondary shrubby woodland at an altitude of between 1200 and $3200 \mathrm{~m}$ [2]. It is a typical distylous species with reciprocally placed stigma and anthers in each floral morph [3]. Nevertheless, our continued field investigations showed that it is now difficult to find wild populations as appropriate habitats have been fragmented due to human activities.

Considering its high ornamental value, endemism and endangered status, we developed 24 microsatellite markers from L. yunnanensis to obtain its genetic information for further studies on conservation of resources, selecting parental types in cross-breeding, as well as evolution of this species at the molecular level.

\section{Results and Discussion}

A total of 317 putative SSR-positive clones were captured, among these 165 clones (52\%) were found to contain simple sequence repeats (SSR). Finally, 48 sequences contained SSR loci were selected for primer design. Twenty-four microsatellite loci successfully amplified in L. yunnanensis for 48 microsatellite loci and 11 of them were polymorphic amplification, other 13 microsatellite loci were monomorphic by the result of polyacrylamide gel (Table 1).

Table 1. Characteristics of 24 microsatellite loci successfully amplified in Luculia yunnanensis.

\begin{tabular}{|c|c|c|c|c|c|}
\hline Locus & Primer sequence $\left(5^{\prime}-3^{\prime}\right)$ & Repeat motif & $\begin{array}{l}\text { Size } \\
\text { (bp) }\end{array}$ & $\begin{array}{l}\mathrm{Ta} \\
\left({ }^{\circ} \mathbf{C}\right)\end{array}$ & $\begin{array}{c}\text { GenBank } \\
\text { Accession No. }\end{array}$ \\
\hline \multirow{2}{*}{ MH $01 *$} & F: GCACTGGCTTAGTTCTTT & \multirow{2}{*}{ (CT) $12(\mathrm{CCCT}) 3(\mathrm{CT}) 4$} & \multirow{2}{*}{213} & \multirow{2}{*}{60} & \multirow{2}{*}{ JN871296 } \\
\hline & R: TGTTTGTCTCAGGGGTAT & & & & \\
\hline \multirow{2}{*}{ MH $02 *$} & F: AAACGAGCAGAAACTGGA & \multirow{2}{*}{ (TG)11(AG)9 } & \multirow{2}{*}{153} & \multirow{2}{*}{60} & \multirow{2}{*}{ JN871297 } \\
\hline & R: GCATTGTCCAACCCTTTA & & & & \\
\hline \multirow{2}{*}{ MH $03 *$} & F: CAACTTCCCAAATTCTGC & \multirow{2}{*}{$(\mathrm{CT}) 16$} & \multirow{2}{*}{322} & \multirow{2}{*}{61} & \multirow{2}{*}{ JN871298 } \\
\hline & R: TACCCAACACTTTCTCACCA & & & & \\
\hline \multirow{2}{*}{ MH $04 *$} & F: TTTCATTCCGTAACCTT & \multirow{2}{*}{$(\mathrm{CT}) 33$} & \multirow{2}{*}{198} & \multirow{2}{*}{57} & \multirow{2}{*}{ JN871299 } \\
\hline & R: GCCAAAAACTTGTATAATTC & & & & \\
\hline \multirow{2}{*}{ MH $05 *$} & F: TCGTACAATTTTGTTGCTCT & \multirow{2}{*}{ (TG)12 } & \multirow{2}{*}{130} & \multirow{2}{*}{61} & \multirow{2}{*}{ JN871300 } \\
\hline & R: CATTCCTCAATAAACCCATA & & & & \\
\hline \multirow{2}{*}{ MH $06 *$} & F: CTCTATCTGTCGCATTCTTC & \multirow{2}{*}{ (TC)19 } & \multirow{2}{*}{201} & \multirow{2}{*}{61} & \multirow{2}{*}{ JN871301 } \\
\hline & R: TCTAGTAATACTATCCTCCACC & & & & \\
\hline
\end{tabular}


Table 1. Cont.

\begin{tabular}{|c|c|c|c|c|c|}
\hline Locus & Primer sequence $\left(5^{\prime}-3^{\prime}\right)$ & Repeat motif & $\begin{array}{l}\text { Size } \\
\text { (bp) }\end{array}$ & $\begin{array}{c}\text { Ta } \\
\left({ }^{\circ} \mathbf{C}\right) \\
\end{array}$ & $\begin{array}{c}\text { GenBank } \\
\text { Accession No. }\end{array}$ \\
\hline \multirow{2}{*}{ MH $07 *$} & F: ACCTCGGATAGAAAACAGC & \multirow{2}{*}{$(\mathrm{GA}) 4(\mathrm{CA}) 6(\mathrm{GA}) 14$} & \multirow{2}{*}{284} & \multirow{2}{*}{57} & \multirow{2}{*}{ JN871302 } \\
\hline & R: GTCGTATATAAAGTAAAGCATTGCC & & & & \\
\hline \multirow{2}{*}{ MH $08 *$} & F: TGAACTCCCCAGTCCCCACT & \multirow{2}{*}{ (AG)22 } & \multirow{2}{*}{187} & \multirow{2}{*}{62} & \multirow{2}{*}{ JN871303 } \\
\hline & R: TCGGTCCACAATCATCACAAAA & & & & \\
\hline \multirow{2}{*}{ MH $09 *$} & F: TGGTCATCAACTATTTTCCCTC & \multirow{2}{*}{ (TC)14 } & \multirow{2}{*}{153} & \multirow{2}{*}{57} & \multirow{2}{*}{ JN871304 } \\
\hline & R: ATGTATTCATTCAGGTATAGAGAGA & & & & \\
\hline \multirow{2}{*}{ MH $10 *$} & F: GTCCTCCATAGCCACAAA & \multirow{2}{*}{$(\mathrm{GAA}) 13$} & \multirow{2}{*}{130} & \multirow{2}{*}{59} & \multirow{2}{*}{ JN871305 } \\
\hline & R: TACTATAACTGATTTTCAAATTTCC & & & & \\
\hline \multirow{2}{*}{ MH $11 *$} & F: GTCCTAGTGTACTTACCCATCA & \multirow{2}{*}{$(\mathrm{CA}) 14$} & \multirow{2}{*}{192} & \multirow{2}{*}{61} & \multirow{2}{*}{ JN871306 } \\
\hline & R: GACAGCAATGCTCCGACT & & & & \\
\hline \multirow{2}{*}{ MH 12} & F: CAATTCTTGAGCCACTTT & \multirow{2}{*}{$(\mathrm{GA}) 13$} & \multirow{2}{*}{208} & 56 & IN871307 \\
\hline & R: GCTTCTGCCAATGTTTAG & & & 56 & JN8/130/ \\
\hline MH 13 & F: AAGAAGGTGAAGGAGGCT & & 262 & 49 & IN871308 \\
\hline MH 13 & R: AGATGGTCCCAATGTAGC & $(\mathrm{IC}) / 2(\mathrm{GI}) /(\mathrm{Cl}) 4$ & 262 & 49 & JN8/1308 \\
\hline MH 14 & F: GGGTTCAAGGCTCAGGAC & (GA) 15 & 221 & 49 & IN871309 \\
\hline MH 14 & R: CATCCCATTGCCAACATC & (UA)12 & 221 & 49 & JN8/1309 \\
\hline MH 15 & F: TATGCTTGGGCAGGAATG & $(G A) 21$ & 201 & 54 & IN871310 \\
\hline MH IS & R: AACGCTGTGCTATCACTGTCTT & $(\mathrm{GA}) 21$ & 201 & 54 & JN8/1310 \\
\hline MH 16 & F: TTTTAGTAGGGTTCACCG & (CT) 32 & 192 & 54 & JN871311 \\
\hline MH 10 & R: CCCACTCCTGGATGTTTG & $(C 1) 32$ & & & \\
\hline MH 17 & F: ATTCTGTATCTGGCTCAC & (CT)14(CA)11 & 279 & 55 & IN871312 \\
\hline MH I / & R: GTCTTTCAAGCATAAAGA & (C1)14(CA)11 & 219 & 55 & JN8/1312 \\
\hline MH 18 & F: ACGAGCAGAAACTGGAAA & (GT)11(GA)9 & 153 & 58 & IN871313 \\
\hline & R: GCATTGTCCAACCCTTTA & & & & \\
\hline MH 19 & F: TCTACTTCGGTTCAGGGTT & $(\mathrm{TC}) 25(\mathrm{AC}) 5$ & 283 & 61 & IN871314 \\
\hline & R: GCTTCCGCTGTAATGGTT & & & & \\
\hline МН 20 & F: ATGACTCCACATAGCAAACA & $(\Delta G) 20$ & 233 & 63 & IN871315 \\
\hline & R: CTGGGCTGATCCTAAACA & $(A G) 20$ & & & \\
\hline МН 21 & F: GGGAAATGGTTCTTTATGGT & (CT) 21 & 142 & 59 & IN871316 \\
\hline MH 21 & R: TCATCATGGGTTGGGTTAT & (C1) 21 & 142 & 59 & JN871316 \\
\hline МH 22 & F: CATCAACTCACGATTGCA & (TA) $6(\mathrm{GA}) 16$ & 236 & 55 & IN871317 \\
\hline $1 \mathrm{M} \Pi 22$ & R: CGTTATGTTTCTTTTCTCCC & $(1 \mathrm{~A}) \mathrm{O(UA)} 10$ & 250 & 35 & JNO $/ 151 /$ \\
\hline МН 23 & F: GCAGGTGAGAATGCAAAT & (AG)27(TG)4 & 157 & 59 & IN871318 \\
\hline & R: AAGCAGCATCACAGTTCC & $(A U) 2 /(I U)^{4}$ & & 39 & JNO/1518 \\
\hline МН 24 & F: GCTTGTTTTGATGGTTGT & (CA)13(GA)25 & 424 & 55 & IN871319 \\
\hline & R: TAGGCGAATGATGCTTAT & & & & \\
\hline
\end{tabular}

* Displayed polymorphisms in Luculia yunnanensis; Ta, PCR annealing temperature.

The number of alleles ranged from two to four in 24 individuals of the species sampled from the two natural populations. Values for $H_{\mathrm{O}}$ and $H_{\mathrm{E}}$ ranged from 0.000 to 0.833 and from 0.431 to 0.771 , with averages of 0.389 and 0.614 , respectively (Table 2). 
These microsatellite markers developed in our study will be a useful tool for further studies of conservation genetics, and will help us understand the genetic structure of L. yunnanensis, so as to make effective conservation strategy for this endangered plant.

Table 2. Results of 11 polymorphic microsatellite loci screening in two populations of Luculia yunnanensis.

\begin{tabular}{ccccccc}
\hline & \multicolumn{3}{c}{ Population 1 $(\boldsymbol{N}=\mathbf{1 2})$} & \multicolumn{3}{c}{ Population 2 $(\boldsymbol{N}=\mathbf{1 2})$} \\
\hline Locus & $N_{\mathrm{A}}$ & $H_{\mathrm{O}}$ & $H_{\mathrm{E}}$ & $N_{\mathrm{A}}$ & $H_{\mathrm{O}}$ & $H_{\mathrm{E}}$ \\
MH 01 & 4 & 0.833 & 0.684 & 3 & 0.666 & 0.489 \\
MH 02 & 2 & 0.333 & 0.463 & 3 & 0.250 & 0.539 \\
MH 03 & 3 & 0.000 & 0.652 & 4 & 0.000 & 0.681 \\
MH 04 & 3 & 0.250 & 0.561 & 3 & 0.250 & 0.684 \\
MH 05 & 4 & 0.416 & 0.655 & 4 & 0.583 & 0.771 \\
MH 06 & 3 & 0.166 & 0.681 & 4 & 0.500 & 0.753 \\
MH 07 & 2 & 0.333 & 0.463 & 5 & 0.583 & 0.768 \\
MH 08 & 3 & 0.166 & 0.594 & 4 & 0.500 & 0.699 \\
MH 09 & 4 & 0.250 & 0.587 & 4 & 0.583 & 0.655 \\
MH 10 & 2 & 0.250 & 0.431 & 2 & 0.666 & 0.463 \\
MH 11 & 3 & 0.666 & 0.648 & 3 & 0.333 & 0.594 \\
\hline
\end{tabular}

$N$, population sample size; $N_{\mathrm{A}}$, number of alleles revealed; $H_{\mathrm{O}}$, observed heterozygosity; $H_{\mathrm{E}}$, expected heterozygosity.

\section{Experimental Section}

Genomic DNA samples of L. yunnanensis were extracted from silica-gel-dried leaves using a modified CTAB methodology [4]. The extracted DNA was dissolved in $30 \mu \mathrm{L}$ TE buffer. A microsatellite enriched library was then conducted using a modified biotin-streptavidin capture method [5]. Total genomic DNA (approximate 250-400 ng) was completely digested with $2.5 \mathrm{U}$ of MseI restriction enzyme (New England Biolabs, Beverly, MA, USA), and then ligated to an MseI AFLP adaptor (5'-TAC TCA GGA CTC AT-3'/5'-GAC GAT GAG TCC TGA G-3') using T4 DNA ligase (New England Biolabs, Beverly, MA, USA). The digested-ligated fragments were diluted in a ratio of $1: 10$, and $5 \mu \mathrm{L}$ of them were used amplification reaction with adaptor-specific primers (5'-GAT GAG TCC TGA GTA AN-3'/5'-TTA CTC AGG ACT CAT CN-3'). The amplified DNA fragments $(200-800 \mathrm{bp})$ were enriched by magnetic bead selection with a 5-biotinylated [(AG) $)_{15}$, $(\mathrm{AAG})_{10}$ and $(\mathrm{AC})_{15}$ ] probe, respectively [6]. The Recovered DNA fragments were reamplified with MseI-N primers. The purified PCR products using EZNA Gel Extraction Kit (Omega Bio-Tek, Guangzhou, China), were ligated into pMD18-T vector (Takara, Dalian, Liaoning, China), and then transformed into DH5a competent cells (Tiangen, Beijing, China). The positive clones were tested using vector primers $\mathrm{T} 3 / \mathrm{T} 7$ and primer $(\mathrm{AC})_{10} /(\mathrm{AG})_{10} /(\mathrm{AAG})_{7}$ respectively. All these PCR reactions had the same conditions: $95{ }^{\circ} \mathrm{C}$ for $3 \mathrm{~min}$ followed by 32 cycles at $94{ }^{\circ} \mathrm{C}$ for $45 \mathrm{~s}, 52{ }^{\circ} \mathrm{C}$ for $1 \mathrm{~min}$, $72{ }^{\circ} \mathrm{C}$ for $1 \mathrm{~min}$, and a final extension step at $72{ }^{\circ} \mathrm{C}$ for $10 \mathrm{~min}$. The positive clones were captured for sequencing with an ABI PRISM 3730XL DNA sequencer (Applied Biosystems, Foster City, CA, USA). Sequences contained simple sequence repeat and enough flanking regions were selected for primer design using Primer Premier 5.0 program [7]. 
The designed Primer pairs were tested in 24 wild individuals of L. yunnanensis from two natural populations collected in Southwest China: population 1 (Lushui County, 26 ${ }^{\circ} 29^{\prime} \mathrm{N}, 98^{\circ} 50^{\prime} \mathrm{E}, 1772 \mathrm{a} . \mathrm{s} .1$.) and population 2 (Fugong County, 25 $58^{\prime} \mathrm{N}, 98^{\circ} 47^{\prime} \mathrm{E}, 1884 \mathrm{a} . \mathrm{s} .1$.). Herbarium voucher deposited in Kunming Institute of Botany, Chinese Academy of Sciences. The PCR amplification was carried out in a total volume of $20 \mu \mathrm{L}$ reaction containing $10 \mu \mathrm{L} 2 \times$ Taq PCR MasterMix (Tiangen; 0.1 U Taq Polymerase/ $\mu \mathrm{L}, 0.5 \mathrm{mM}$ dNTP each, $20 \mathrm{mM}$ Tris- $\mathrm{HCl}(\mathrm{Ph} 8.3), 100 \mathrm{mM} \mathrm{KCl}, 3 \mathrm{mM} \mathrm{MgCl}$ ), $0.5 \mu \mathrm{L}$ of each primer and 50-100 ng Genomic DNA. PCR amplifications were conducted under the following conditions: $95{ }^{\circ} \mathrm{C}$ for $5 \mathrm{~min}$ followed by 32-35 cycles at $94{ }^{\circ} \mathrm{C}$ for $45 \mathrm{~s}$, at the annealing temperature for each specific primer (optimized for each locus, Table 1) for $45 \mathrm{~s}, 72{ }^{\circ} \mathrm{C}$ for $1 \mathrm{~min}$, and a final extension step at $72{ }^{\circ} \mathrm{C}$ for $10 \mathrm{~min}$. PCR products were separated on $8 \%$ polyacrylamide denaturing gel using a $20 \mathrm{bp}$ ladder molecular size standard by silver staining.

The data was analyzed by GENEPOP 4.0 [8], which included test of observed heterozygosity $\left(H_{O}\right)$ and expected heterozygosity $\left(H_{E}\right)$ for the 11 polymorphic microsatellite loci.

\section{Conclusions}

In summary, 24 microsatellite markers have been specifically developed for L. yunnanensis in this study. These markers will facilitate further studies on the population genetics of L. yunnanensis and its allied species. They are also expected to be useful for parental selections in controlled hybridization breeding programs and enable us to protect sources of germplasm through genetic diversification via ex situ nurseries and reintroduction programs.

\section{Acknowledgements}

This work was supported by the Special Fund of Basic Research of Central Nonprofit Research Institutes (Riricaf 201003M).

\section{References}

1. Chen, W.Q. Flora of Yunnan (Chinese Version); Science Press: Beijing, China, 2003; Volume 15, p. 20.

2. Luo, X.R.; Gao, W.Z.; Chen, W.Q.; Xu, X.H.; Wu, H. Flora of China (Chinese Version); Science Press: Beijing, China, 1999; Volume 71, p. 238.

3. Zhou, W.; Wang, H.; Li, D.Z.; Yang, J.B. Isolation and characterization of 13 microsatellite loci from Luculia pinceana (Rubiaceae), a typical distylous species. Hortscience 2010, 45, 840-841.

4. Doyle, J.J.; Doyle, J.L. A rapid DNA isolation procedure for small quantities of leaf tissue. Phytochem. Bull. 1987, 19, 11-15.

5. Chen, T.; Zhou, R.C.; Ge, X.J.; Shi, S. Development and characterization of microsatellite markers for a mangrove tree species Sonneratia caseolaris (L.) Engler (Lythraceae sensu lato). Conserv. Genet. 2008, 9, 957-959.

6. Zane, L.; Bargelloni, L.; Patarnello, T. Strategies for microsatellite isolation: A review. Mol. Ecol. 2002, 11, 1-16. 
7. Clarke, K.R.; Gorley, R.N. PRIMER (Version 5): User Manual/Tutorial; PRIMER-E: Plymouth, UK, 2001; p. 91.

8. Raymond, M.; Rousset, F. GENEPOP (version 1.2): Population genetics software for exact tests and ecumenicism. J. Hered. 1995, 86, 248-249.

(C) 2012 by the authors; licensee MDPI, Basel, Switzerland. This article is an open access article distributed under the terms and conditions of the Creative Commons Attribution license (http://creativecommons.org/licenses/by/3.0/). 